\title{
High-resolution computed tomography in Mycobacterium avium complex disease: one step forward, two steps back
}

\section{To the Editors:}

We read with interest the article by KUROISHI et al. [1], in which the group pleasantly documented the prognostic implications of high-resolution computed tomography (HRCT) findings in $\mathrm{Myco-}$ bacterium avium complex (MAC) disease. KuROISHI et al. [1], found that the presence of atelectasis, cavities, pleural thickening or bronchiectasis on a computed tomography (CT) scan indicated poor prognosis and concluded that CT findings are good predictors of the response to treatment in MAC disease patients. However, there are a few practical issues regarding the topic that need to be addressed.

Although HRCT requires less radiation, a chest CT scan is associated with radiation exposure of $8 \mathrm{mSv}$, equivalent to a radiation dose of 400 chest radiographs [2]. Exposing already immunocompromised patients of nontuberculous mycobacterial (NTM) infection to high dose radiation to predict the treatment outcome does not sound justified. Moreover, it is also associated with serious health hazards, notably an increased risk of cancer [3]. Thus, the risk-benefit ratio in such cases needs to be assessed. It also needs to be emphasised that the practice of using CT scans for such indications may inadvertently increase the irrational use of CT scans.

The radiological presentation of pulmonary infection by NTM is nonspecific and overlaps considerably with pulmonary tuberculosis [4]. Moreover, NTM disease has a predilection to occur in architecturally distorted lungs of old, treated, pulmonary tuberculosis patients. Therefore, judging the prognosis on the basis of CT findings alone may not present a true picture. Moreover, the cost of performing a CT scan is also of great concern, especially in developing countries such as India, where the burden of infection is substantial and the cost of a CT scan is beyond the reach of many people.

Finally, the total duration of treatment (minimum 12 months) mentioned in the study by KuROISHI et al. [1], is not in accordance with the American Thoracic Society guidelines, which recommends that treatment should continue for $1 \mathrm{yr}$ of consecutively negative sputum cultures [5]. This delivers the wrong message to the readers and raises questions on the validity of the study's findings, as the authors have taken consecutive negative sputum cultures over a period of 3 months (not the recommended 12 months) as the gold standard for the parameter concerning the treatment outcome for comparison.

We feel that the treatment outcome in cases of nontuberculous mycobacterial infection should be predicted by comprehensively evaluating the severity of clinical features at initial presentation, presence of comorbidities, sputum smear grading of acid fast bacilli and radiological findings. High-resolution computed tomography should only be performed in selected cases, where there is a difficulty in diagnosing the disease or the response to treatment is unsatisfactory.

\section{Aggarwal and P.R. Mohapatra}

Pulmonary Medicine, Government Medical College and Hospital, Chandigarh, Punjab, India.

\section{STATEMENT OF INTEREST}

None declared.

\section{REFERENCES}

1 Kuroishi S, Nakamura Y, Hayakawa H, et al. Mycobacterium avium complex disease: prognostic implication of highresolution computed tomography findings. Eur Respir J 2008; 32: 147-148.

2 Picano E. Sustainability of medical imaging. BMJ 2004; 328 : 578-580.

3 Brenner DJ, Elliston CD. Estimated radiation risks potentially associated with full-body CT screening. Radiology 2004; 232: 735-738.

4 Koh WJ, Yu CM, Suh GY, et al. Pulmonary TB and NTM lung disease: comparison of characteristics in patients with AFB smear-positive sputum. Int J Tuberc Lung Dis 2006; 10: 1001-1007.

5 Griffith DE, Aksamit T, Brown-Elliott BA, et al. An official ATS/IDSA statement: diagnosis, treatment, and prevention of nontuberculous mycobacterial diseases. Am J Respir Crit Care Med 2007; 175: 367-416.

DOI: 10.1183/09031936.00070608

\section{From the authors:}

We would like to thank D. Aggarwal and P.R. Mohapatra for their interest in our article [1]. In their letter, D. Aggarwal and P.R. Mohapatra suggest that the risk/benefit ratio in nontuberculous mycobacteria (NTM) patients needs to be assessed, since high-dose radiation is associated with serious health hazards. In addition, the cost of performing a computed tomography (CT) scan is also of great concern. We agree that the indication of CT for NTM patients needs to be assessed. However, the usefulness of CT for the diagnosis of Mycobacterium avium complex disease is obvious in many previous reports [2-4]. Although we explained the risk of radiation hazards to the patients, most of them agreed to have a CT examination. They also suggest that in previously treated pulmonary tuberculosis patients, judging the prognosis solely on the basis of CT findings may not present a true picture. We 
agree with this comment, and in our study we had excluded the patients with healed tuberculosis as described in the materials and methods section [1]. The final comment from D. Aggarwal and P.R. Mohapatra is that the duration of treatment is not in accordance with the American Thoracic Society/Infectious Disease Society of America statement [4]. The treatment could have been continued until 1-yr of consecutive sputum culture negativity was established. We had basically followed this recommendation and in our study, the duration of treatment after sputum conversion was $14.2 \pm 3.2$ months. D. Aggarwal and P.R. Mohapatra feel the treatment outcome of a NTM infection should be predicted by evaluating the severity of clinical features, presence of comorbidities, sputum smear grading of acid fast bacilli and radiological findings.

We agree with this comment; however, we think those factors should also be scientifically demonstrated as prognostic markers in future studies.

\section{S. Kuroishi and Y. Nakamura}

Second Division, Dept of Internal Medicine, Hamamatsu University School of Medicine, Shizuoka, Japan.

\section{STATEMENT OF INTEREST}

None declared.

\section{REFERENCES}

1 Kuroishi S, Nakamura Y, Hayakawa H, et al. Mycobacterium avium complex disease: prognostic implication of highresolution computed tomography findings. Eur Respir $J$ 2008; 32: 147-152.

2 Tanaka E, Amitani R, Niimi A, Suzuki K, Murayama T, Kuze F. Yield of computed tomography and bronchoscopy for the diagnosis of Mycobacterium avium complex pulmonary disease. Am J Respir Crit Care Med 1997; 155: 2041-2046.

3 Jeong YJ, Lee KS, Koh WJ, Han J, Kim TS, Kwon OJ. Nontuberculous mycobacterial pulmonary infection in immunocompetent patients: comparison of thin-section CT and histopathologic findings. Radiology 2004; 231: 880-886.

4 Griffith DE, Aksamit T, Brown-Elliott BA, et al. An official ATS/IDSA statement: diagnosis, treatment, and prevention of nontuberculous mycobacterial diseases. Am J Respir Crit Care Med 2007; 175: 367-416.

\section{COPD and osteoporosis: a lot of work to be done}

\section{To the Editors:}

There is no doubt that on reading what is suggested by FABBRI $e t$ al. [1] on the view of chronic obstructive pulmonary disease (COPD), a holistic vision of the patient turns out to be extremely absorbing and a cornerstone in our medical grounding.

However, as often happens, reality far exceeds expectations. In a retrospective analysis carried out on 885 outpatients (507 males and 378 females) treated at our pneumatological outpatient clinic over the last 2 yrs, 297 (178 males and 119 females) had COPD; of whom only $10(1.13 \%)$ were diagnosed as suffering from osteoporosis. In all other cases, the necessary examination to assess the mineral bone density was not carried out, in spite of a chronic respiratory pathology and an apparent reduction of muscular mass, owing to reduction in physical exertion, and notwithstanding the steroid therapy. The number of subjects diagnosed is thus a dramatically low percentage, despite the abundance of literature now available. According to the guidelines of the Italian Health Department on osteoporosis [2], prevention of fractures is an objective aimed at improving the quality of life, assisting drastic reduction in social costs and obtaining health economy. With a view to gaining valid prevention of osteoporosis at all ages, the key steps to follow could be the following: stick to a diet rich in calcium and vitamin D; exert physical exercise proportionate to body weight; define mineral bone density; and undergo the correct therapy [3-6]. Aside from our 10 outpatients treated for osteoporosis, no preventive strategy was planned for the remaining patients, nor any stratification of the risk of loss of bone mass was executed. Our conclusions are valid for the sampled patients, but we cannot say whether they can be extended to all patients with COPD. In our view, it is crystalclear that physicians must be aware of the effects on bone mass related to COPD, as well as those of the chronic administration of steroids or due to consequent inaction or smoking. We cannot believe that "the fact that chronic diseases seldom occur in isolation is still largely ignored" [1]. Nevertheless, this analysis proves the lack of preventive intervention in osteoporosis. A reason for this situation could be the economic drift of our public health system and, as a result, the consequent goal is to reach a balanced budget, rather than attain the planned final outcome, irrespective of the self-evident collateral effects and long-term relapses.

To the public health system, the cost per patient for 1-yr of treatment with biphosphonate ranges between $€ 292.00$ and $€ 524.00$ [7], which is not a minor burden, when we consider the number of patients with COPD and of all plausible cases of osteoporosis [8].

Moreover, these data suggest that it is necessary for the daily practical clinic to take into account how important the stratification of the risk of secondary osteoporosis is for all patients with chronic pathologies under steroid therapy, as well as the importance of the preventive nonpharmacological treatment.

Furthermore, based upon scientific evidence, more frequent communication becomes mandatory between medical teams on one hand and those who manage the funds on the other, as far as intentions and objectives are concerned.

\section{S. Zeminian, M. Pattarello and F. Rubini}

Rehabilitative Respiratory Unit, Marzana Hospital, Verona, Italy. 